\title{
ULTRAFAST PROCESSES IN SEMICONDUCTOR STRUCTURES
}

\author{
L. VIÑA \\ Departamento de Física de Materiales C-IV, Universidad Autónoma de Madrid \\ Cantoblanco, 28049 Madrid, Spain
}

\begin{abstract}
We review the dynamics of some of the more relevant optical processes in semiconductor quantum wells. We concentrate on the linear regime and study the time evolution of the light emission, using time-resolved photoluminescence spectroscopy. In intrinsic materials, excitonic effects determine their optical properties. Here we describe the formation and recombination of excitons, and the dependence of these processes on lattice temperature, exciton density, and energy of the excitation light pulses. We also describe the dynamics of the exciton's spin by optical orientation experiments. We discuss the principal mechanisms responsible for the spin flip of the excitons and clarify the role of the exciton localization. Finally, we will show that exciton-exciton interaction produces a breaking of the spin degeneracy in two-dimensional semiconductors. In doped quantum wells, we show that the two spin components of an optically created two-dimensional electron gas are well described by the Fermi-Dirac distributions with a common temperature but different chemical potentials. The rate of the spin depolarization of the electron gas is found to be independent of the mean electron kinetic energy but accelerated by thermal spreading of the carriers.
\end{abstract}

PACS numbers: 71.35.Cc, 71.35.Lk, 71.70.Gm, 78.47.+p, 78.55. $\mathrm{Cr}$

\section{Introduction}

The study of exciton dynamics in semiconductor quantum wells (QW's) has attracted an intense interest in recent years. A large number of studies have focused on the dynamics of exciton formation and relaxation [1-12]. It is generally accepted that the relaxation process following the optical creation of an electron-hole pair in the continuum may be separated into two steps [3]: first, in a time of the order of tens of picoseconds, the electron-hole pair relaxes its energy and forms an exciton with high kinetic energy. In the second step, which can last up to hundreds of picoseconds, the excess energy is lost, within the $1 s$ excitonic branch, in a slow process that brings the exciton to states with center of mass momentum $(\boldsymbol{K})$ near zero, where the exciton may recombine emitting a photon.

Despite this agreement in the fundamental ideas of exciton formation and relaxation, there are several aspects of these processes which are still controversial. 
Some authors have found that exciton dynamics in GaAs QW's are independent of excitation energy, even for excitation at the light-hole (lh) exciton energy, or exciting at one LO phonon above the detection energy [3]. However, in similar systems, other works have shown a significant slowing down of the relaxation process when exciting at the lh exciton energy [6] and have even demonstrated an oscillation in the exciton luminescence rise time as a function of excitation energy [7]. This oscillation has been interpreted as the occurrence of a selective optical-phonon assisted exciton formation, suggesting that the exciton formation process from free electrons and holes is sensitive to the wave vector of the electrons and holes after the initial LO-phonon cascade [7].

Useful electronic devices rely on the precise control of electronic charge, and in general the fact that the electrons also have a spin is ignored. However, the scattering processes for electrons depend on their spin state. There are various experiments to detect optical orientation, such as spin polarization of electron photoemission, electron paramagnetic resonance, nuclear magnetic resonance, spin-dependent transport, the Faraday rotation, and spin-dependent pump-and-probe or recombination. The Hanle effect [13], i.e. the spin depolarization of carriers subject to a transverse magnetic field, has been traditionally used to obtain quantitative values for the spin relaxation times. Optical pumping, with linearly polarized light, and orientation, with circularly polarized light, of carrier spins are powerful methods to investigate relaxation processes in semiconductors and have also found applications in spin-polarized electron sources. With the appearance of ultrafast lasers, the spin relaxation time can be also measured in a direct way. The emitted luminescence, after an excitation with a light pulse, reflects the temporal evolution of the carrier distribution and can be analyzed in polarization to study energy as well as orientation relaxation rates.

Experimental investigations on the spin dynamics in low-dimensional semiconductors have flourished in the last decade. Many works deal with the spin processes of excitons [14-22], including the study of the influence of external electric fields [23]. Fewer investigations deal with the spin flip of individual electrons and holes in 2D systems [16, 17, 24-26]. Extensive theoretical studies have been also done on the spin-flip relaxation of excitons [27-30] and free carriers [31-34].

In this paper we will concentrate on the study of the time evolution of the light emission, using time-resolved photoluminescence (TR-PL) spectroscopy, in intrinsic and doped QW's. We will pay special attention to phenomena related with the spin of the carriers and the excitons. The rest of the manuscript is organized as follows. Section 2 gives the experimental details. The exciton dynamics is presented in Sec. 3. The excitonic spin relaxation is shown in Secs. 4 and 5. The dynamics of a spin polarized, optically pumped electron gas is presented in Sec. 6. Finally, we summarize in Sec. 7.

\section{Experimental details}

The experiments are performed in a temperature variable, cold finger cryostat exciting the samples with light pulses. These are obtained from either a mode-locked Nd-YAG laser, which synchronously pumps a cavity-matched dye laser, or a $\mathrm{Ti}$ : sapphire mode-locked laser pumped by an $\mathrm{Ar}^{+}$-ion laser. The pulse 
width are $5 \mathrm{ps}$ and $1.2 \mathrm{ps}$ for the dye and the $\mathrm{Ti}$ : sapphire systems, respectively. The incident light is directed along the growth axis of the heterostructures and a backscattering geometry is used. The PL is time-resolved in a standard up-conversion spectrometer. The time resolution, obtained by overlapping on a nonlinear crystal, $\mathrm{LiIO}_{3}$, of the luminescence from the sample with a delayed pulse from the laser, is basically determined by the temporal pulse width. A double grating monochromator is used to disperse the up-converted signal. The exciting light is circularly polarized by means of a $\lambda / 4$ plate, and the PL is analyzed into its $\sigma^{+}$ and $\sigma^{-}$components using a second $\lambda / 4$ plate before the nonlinear crystal. Time delays at a given emission energy or time-resolved PL spectra at different delays after the excitation pulse are obtained using this system.

For the dynamics of excitons we concentrate on the results of a GaAs/AlAs multiquantum well, consisting of 50 periods of nominally $77 \AA$ wide GaAs wells and $72 \AA$ wide AlAs barriers. The sample is exceptionally bright and presents a small Stokes shift, which allows us to perform quasi-resonant excitation at the free heavy-hole exciton, observed in pseudo-absorption experiments (PL excitation), detecting at the weakly-bound exciton seen in PL. We also used tensile strained $\mathrm{GaAs}_{1-x} \mathrm{P}_{x} / \mathrm{Al}_{0.35} \mathrm{Ga}_{0.65} \mathrm{As} \mathrm{QW}$ 's, with $\mathrm{P}$ contents of $5 \%, 8 \%$, and $12 \%$. In each $\mathrm{GaAsP} / \mathrm{AlGaAs}$ sample there are several $\mathrm{GaAs}_{1-x} \mathrm{P}_{x} \mathrm{QW}$ 's with different thicknesses, separated by $400 \AA \mathrm{Al}_{0.35} \mathrm{Ga}_{0.65} \mathrm{As}$ barriers.

For the measurements of the electron spin-relaxation, we studied $p$-type modulation doped GaAs/GaAlAs QW's with hole sheet concentrations of $\approx 3 \times 10^{11} \mathrm{~cm}^{-2}$, mobilities of $\approx 4000 \mathrm{~cm}^{2} \mathrm{~V}^{-1} \mathrm{~s}^{-1}$ and well widths from 30 to $80 \AA$. These structures were initially extensively tested with conventional, low power, cw experiments [35]. Here, we will show the results obtained in a $30 \AA$ thick QW modulation-doped with beryllium. We measured PL spectra at fixed times, as well as the PL decay at fixed emission energies, both as a function of temperature $(T=10-50 \mathrm{~K})$ and as a function of the laser power, giving concentrations of excess carriers in the range between $\approx 10^{10} \mathrm{~cm}^{-2}$ and $\approx 10^{11} \mathrm{~cm}^{-2}$.

\section{Exciton dynamics}

Figure 1 shows the cw PL (dark area) and the photoluminescence excitation (PLE) spectra (gray area) of the intrinsic GaAs/AlAs QW. The spectra were recorded at $2 \mathrm{~K}$ under very low excitation density $\left(5 \mathrm{~mW} \mathrm{~cm}^{-2}\right)$. The first peak in both spectra is due to the heavy-hole (hh) exciton. The Stoke shift between the hh exciton peaks in PL and PLE, amounting to $2.5 \mathrm{meV}$, indicates that the PL originates from localized excitons. However, increasing the temperature (not shown) the Stokes shift decreases, and it vanishes at $40 \mathrm{~K}$. The linear temperature dependence of the decay time (see below), which is characteristic of free excitons [1], also indicates that localization effects are not very important. The step at $1.626 \mathrm{eV}$ in the PLE spectra corresponds to the onset of the hh-e intersubband continuum, and the peak at $1.646 \mathrm{eV}$ originates from the light-hole (lh) exciton. Figure 2 illustrates the time evolutions of the total PL (sum of the $\sigma^{+}$and $\sigma^{-}$ components) exciting with $\sigma^{+}$polarized light at two different energies, $1.617 \mathrm{eV}$ filled circles (a), and $1.630 \mathrm{eV}$ - open circles (b). Both sets of time profiles were 

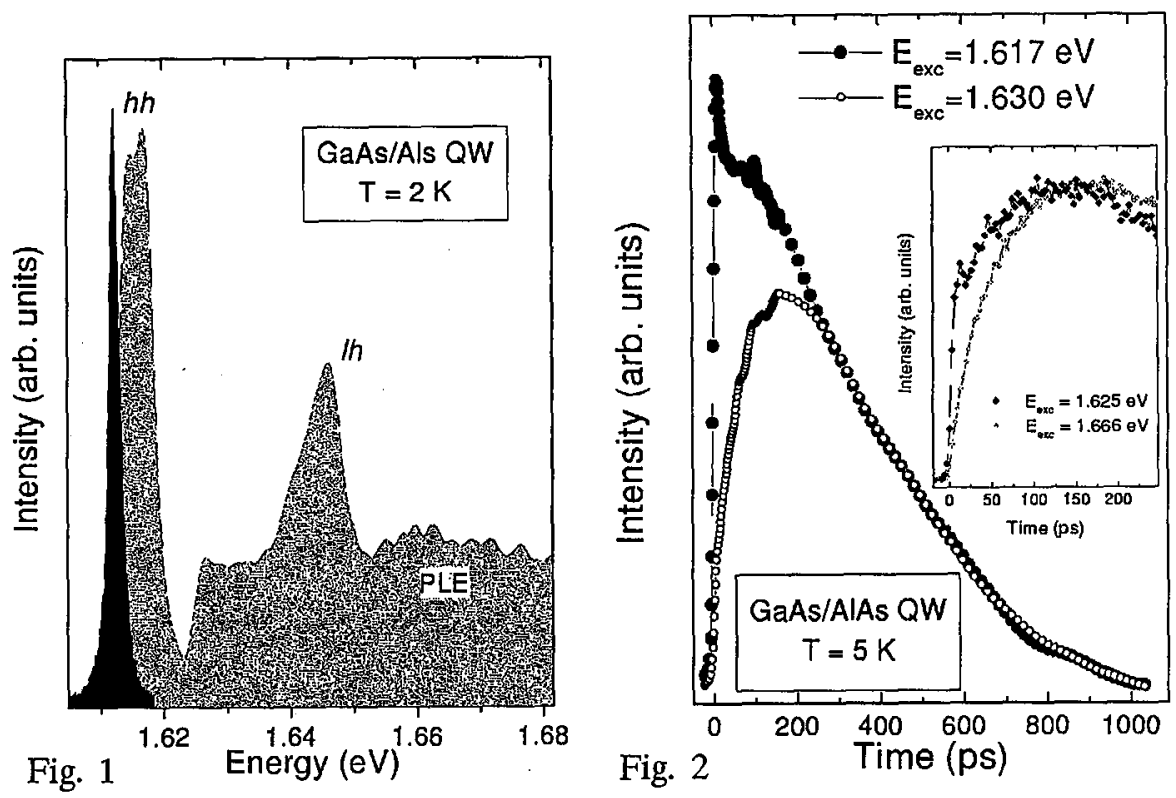

Fig. 1. Photoluminescence (black area) and excitation spectra (gray area) of a $77 \AA$ wide $\mathrm{GaAs}$ recorded at $2 \mathrm{~K}$ under an excitation of $5 \mathrm{~mW} \mathrm{~cm}^{-2}$. The peaks are the heavy-hole (hh) and light-hole (lh) excitons. The step corresponds to the hh subband continuum.

Fig. 2. Time evolution of the PL of the sample shown in Fig. 1, detecting at the peak of the PL and exciting at two different energies. The traces were recorded at $5 \mathrm{~K}$. The inset shows similar traces recorded with excitation at the subband edge (solid points) and with a large excess energy (open points).

recorded detecting the maximum of the PL band $(1.612 \mathrm{eV})$, with an excitation power creating an initial excitonic density of $4 \times 10^{10} \mathrm{~cm}^{-2}$.

The large difference in the rise times of the two curves in Fig. 2 reflects the fact that curve $b$ corresponds to the excitation of electron-hole pairs in the subband continuum ("non-resonant excitation"), and excess energy has to be lost before the exciton can recombine. On the other hand, in the case of curve $a$ the $1 s$-hh excitons have been created resonantly with $\boldsymbol{K}=0$, and they can directly couple to light. The fast initial decay observed in trace $a$ is related to the filling of dark excitonic states due to hole spin flip, and it will be discussed in Sec. 4 .

We observed that for non-resonant excitation, exciton dynamics is independent of excitation energy, even for excitation at the light hole exciton energy, or exciting at one LO phonon above the detection energy. These results are in agreement with the results of previous works [3,4], which have shown that the initial dynamics of non-resonantly excited luminescence is dominated by the slow relaxation process from excitons with large wave vectors to $\boldsymbol{K}=0$ excitons. This slow relaxation process occurs as a consequence of exciton-exciton and exciton-phonon interactions [3]. 
Although our results are in line with the main ideas of the current understanding of exciton relaxation, for excitation energies close to the subband edge we observed a fast initial raise of the luminescence which reveals the existence of a different relaxation process. These results are shown in the inset of Fig. 2, where the filled (open) diamonds depict the time evolution of the total PL exciting with $\sigma^{+}$polarized light at $1.625 \mathrm{eV}(1.666 \mathrm{eV})$. The fast initial raise of the luminescence, for excitation close to the subband edge, indicates that a large number of excitons have reached $\boldsymbol{K}=\mathbf{0}$ states in a time of the order of our time resolution, revealing the existence of a fast relaxation process, which could be related to the relaxation from $n s(n>1)$ excitonic states to $1 s$ states, since it is only observed for excitation energies close to the subband edge.

Further understanding of exciton momentum relaxation processes is obtained from the temperature and carrier-density dependence of the time evolution of the photoluminescence. Figure 3 shows time profiles of the PL, for a carrier density of $4 \times 10^{10} \mathrm{~cm}^{-2}$, at different lattice temperatures. The detection energy was set at the maximum of the PL and a difference of $20 \mathrm{meV}$ between excitation and detection was used for all the temperatures. The data were fitted to a two-level dynamical model which includes two characteristic times: the formation time of excitons with $\boldsymbol{K}=0, \tau_{\mathfrak{r}}$, which corresponds to the rise time in the model, and the exciton recombination time, $\tau_{\mathrm{d}}$, represented by the decay time of the PL. In this model the PL intensity is given by

$$
I(t)=N_{0} \frac{1}{\tau_{\mathrm{r}}-\tau_{\mathrm{d}}}\left[\exp \left(-t / \tau_{\mathrm{r}}\right)-\exp \left(-t / \tau_{\mathrm{d}}\right)\right]
$$

where $N_{0}$ is the initial population of the excitons with $\boldsymbol{K}>\mathbf{0} ; 1 / \tau_{\mathrm{r}}$ is the rate of relaxation of the excitons from the initial state to the $\boldsymbol{K}=\mathbf{0}$ state, from where they recombine. The results of the fits are shown as solid lines and the temperature dependence of $\tau_{\mathrm{r}}$ and $\tau_{\mathrm{d}}$ are presented in the inset of the figure. The decrease in $\tau_{\mathrm{r}}$ with increasing temperature is a consequence of the spreading of the thermalized exciton distribution around $\boldsymbol{K}=\mathbf{0}$ as $T$ increases [3]. Therefore the initial distribution of large- $\boldsymbol{K}$ excitons approaches the final $\boldsymbol{K}=\mathbf{0}$ distribution faster at higher temperatures. Furthermore, the number of excitons at $\boldsymbol{K}=0$ is smaller and consequently the intensity decreases.

The increase in $\tau_{\mathrm{d}}$ with $T$ seems surprising at first view, since a decrease in the excitonic lifetime with increasing temperature is expected. However, taking into account that $\tau_{\mathrm{d}}$ measures the decay of the number of excitons at $\boldsymbol{K}=\mathbf{0}$ and not the excitonic lifetime, the apparent contradiction is solved. When the excitons at rest recombine, those with $\boldsymbol{K}>\mathbf{0}$ fill the states at $\boldsymbol{K}=\mathbf{0}$ and as a consequence the decay of the excitons at rest lasts longer than the lifetime. With increasing $T$ this effect becomes more and more important. With this arguments Feldmann and coworkers have found that, for free excitons, $\tau_{d}$ increases linearly with $T$, while it does not depend on $T$ for bound excitons [1]. The linear dependence of $\tau_{d}$ shown in the inset of Fig. 3 manifests that localization effects are not very important in our sample, specially at the relatively high levels of excitation used for the TR-PL experiments. 


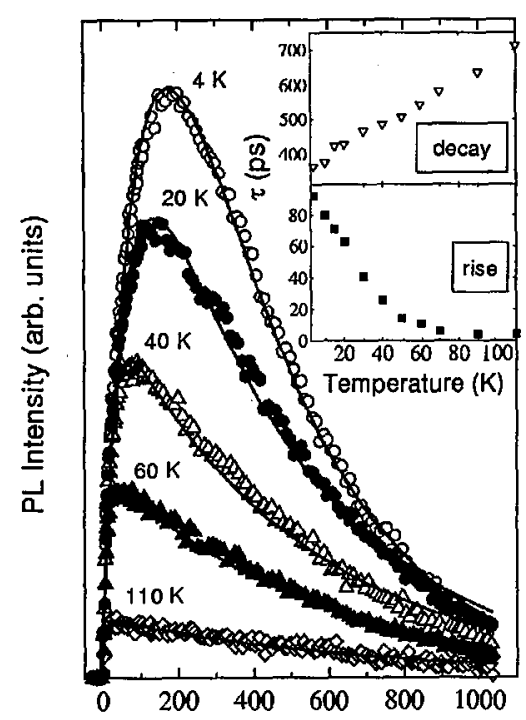

Fig. 3 Time (ps)

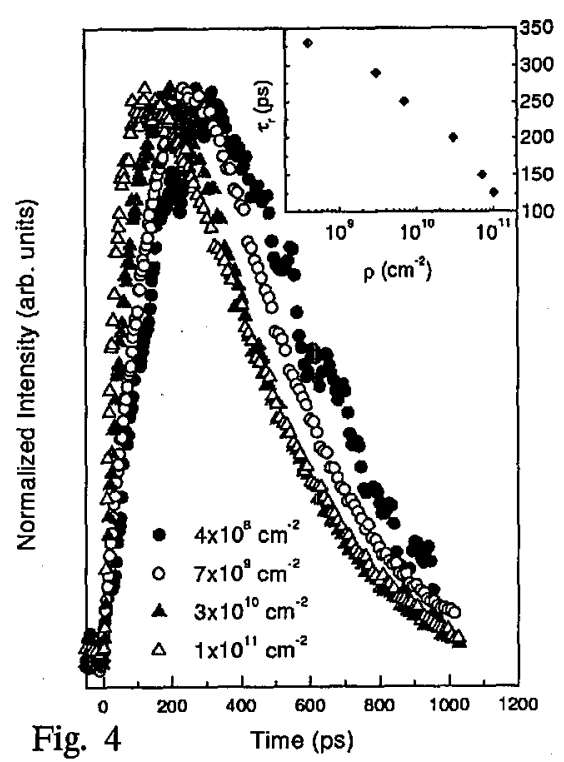

Fig. 3. PL-time evolution of the intrinsic sample for different lattice temperatures. The lines are the results of the fits with a simple dynamical model, obtaining the rise and decay times shown in the insets.

Fig. 4. PL-time evolution of the intrinsic sample for different initial carrier densities. The inset shows the carrier density dependence of the rise time.

The time profiles of the PL, normalized to the maximum intensity, for different carrier densities at $4 \mathrm{~K}$ are depicted in Fig. 4. It is clearly seen that the rise time decreases with increasing density. This behavior agrees with the idea that exciton-exciton collisions are important in the relaxation processes of large- $\boldsymbol{K}$ excitons to $\boldsymbol{K}=\mathbf{0}$ excitons [3].

Let us concentrate now on the results obtained for the $\mathrm{GaAs}_{1-x} \mathrm{P}_{x} /$ $\mathrm{Al}_{0.35} \mathrm{Ga}_{0.65} \mathrm{As} \mathrm{QW}$ 's. In this system, the presence of phosphorus in the well layer decreases the magnitude of the lattice parameter, and the lattice mismatch between the GaAs and the $\mathrm{GaAs}_{1-x} \mathrm{P}_{x}$ is entirely accommodated in the $\mathrm{GaAs}_{1-x} \mathrm{P}_{x}$ layer as a biaxial tensile strain [36]. In response to the biaxial tension, the $\mathrm{GaAs}_{1-x} \mathrm{P}_{x}$ layer relaxes along the growth direction, giving rise to an uniaxial compression. The total strain reduces the energy gap and shifts the heavy hole and light hole valence subbands to higher energies [37]. The amount of band-edge shift of the lh subband is larger than that of the hh one. Thus, the inclusion of strain gives an extra degree of freedom to tune the energy splitting between the lh- and hh-subband edges. Therefore, new subbands structures, including that of degenerate lh- and hh-subband edges, or even of the highest subband being light-hole-like can be achieved. This is shown in Fig. 5, where we depict the PLE spectra for the investigated $\mathrm{GaAs}_{1-x} \mathrm{P}_{x} / \mathrm{Al}_{0.35} \mathrm{Ga}_{0.65} \mathrm{As} \mathrm{QW}$ 's. These QW's present also a Stokes shift of $\approx 5 \mathrm{meV}$. The identification of the heavy or light character of the excitons has been obtained from previous PLE experiments using circular polarization of 
the exciting light and analyzing the polarization of the emission [38]. The smallest peak in each spectra corresponds to the lh exciton, while the largest one to the hh exciton. Those spectra which show only one structure correspond to a quasi-degenerate case. It is clearly seen in the figure that the relative position of the hh and lh excitonic states depend on well thickness $(d)$ and on phosphorus composition $(x)$. The $80 \AA \mathrm{P}=5 \% \mathrm{QW}$ presents the usual configuration, with the hh exciton as the ground excitonic state. The $120 \AA \mathrm{P}=8 \%$ and $120 \AA \mathrm{P}=12 \%$ QW's show an "inverse" configuration, where the ground state is the lh exciton, and the other three wells present a quasi-degenerate hh-lh exciton ground state.

Previous magnetooptical studies performed in these $\mathrm{GaAs}_{1-x} \mathrm{P}_{x} /$ $\mathrm{Al}_{0.35} \mathrm{Ga}_{0.65} \mathrm{As}$ samples have yielded excitonic effective masses, binding energies, and lh-hh subband edge separations [39]. These investigations have demonstrated the flattening of the valence subbands, as the energy difference between the hh and $\mathrm{lh}$ states is decreased, as a result of strain and quantum confinement effects. Therefore, for these tensile strained QW's, the study of the time evolution of the PL will provide information, not only on the effects of the character of the ground excitonic state on exciton dynamics, but also on the influence of the valence band dispersion.

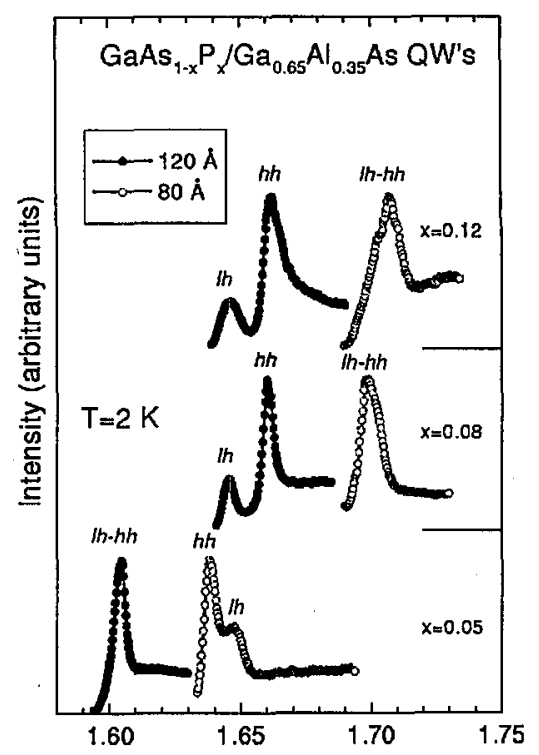

Fig. 5

Energy (eV)

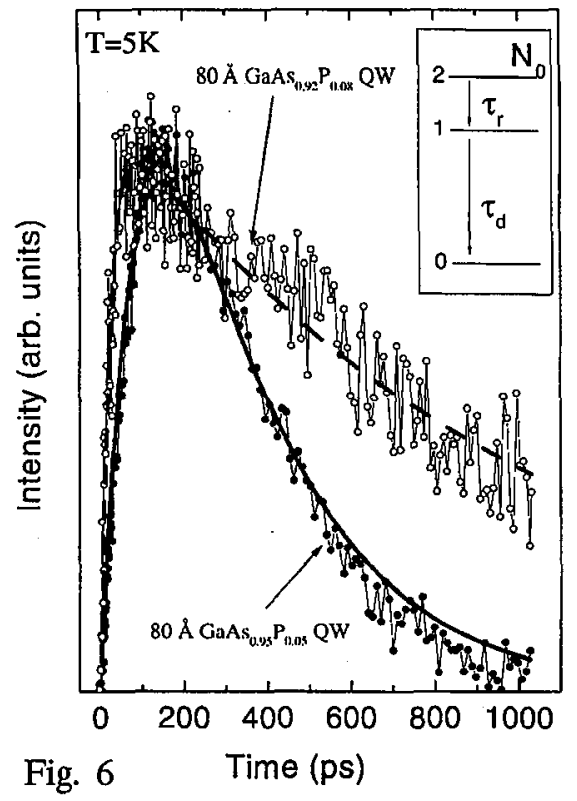

Fig. 5. Photoluminescence excitation spectra, PLE, of six different $\mathrm{GaAs}_{1-x} \mathrm{P}_{x} \mathrm{QW}$ 's. The solid (open) points correspond to the $120 \AA(80 \AA)$ QW's. The spectra were taken at $2 \mathrm{~K}$, exciting with $\sigma^{+}$polarized light and detecting the $\sigma^{-}$polarized light.

Fig. 6. Low-temperature time evolutions of the PL, emitted by the $80 \AA$ GaAs $0.92 P_{0.08}$ and the $80 \AA \mathrm{GaAs}_{0.95} \mathrm{P}_{0.05} \mathrm{QW}$ 's. The intensity has been normalized to its maximum value. The lines are the best fits to the dynamic model shown at the inset figure. 
For the three QW's which correspond to the case of non-degenerate excitonic ground state, the PL rise times are of the order of $140 \mathrm{ps.} \mathrm{This} \mathrm{value} \mathrm{is} \mathrm{similar}$ to the value of the rise time measured in the unstrained GaAs/AlAs system under non-resonant excitation. The long rise time indicates that exciton emission is dominated by the slow relaxation process from large- $\boldsymbol{K}$ excitons to $\boldsymbol{K}=\mathbf{0}$ excitons. However, for the three wells which present a quasi-degenerate hh-lh excitonic ground state, we observed a faster exciton relaxation, with rise times of the order of $60 \mathrm{ps}$. We obtained also that $\tau_{\text {decay }}$ depends on the hh or lh character of the excitonic ground state. QW's having a hh ground state present a decay time of $\approx 500 \mathrm{ps}$, while the value is doubled to $\approx 1 \mathrm{~ns}$ for QW's having a lh state. This behavior is in agreement with the larger value of the oscillator strength of the hh transitions compared to that of the $\mathrm{lh}$ ones.

These results are illustrated in Fig. 6, where the normalized time evolution of the sum of the $\sigma^{+}$and $\sigma^{-}$components of the PL is presented for the $80 \AA \mathrm{P}=8 \% \mathrm{QW}$ (open points), which presents a quasi-degenerate hh-lh excitonic ground state, and for the $80 \AA \mathrm{P}=5 \% \mathrm{QW}$ (solid points), which displays a hh excitonic ground state. Both time evolutions were recorded exciting above the continuum edge with the same excess energy of $60 \mathrm{meV}$. It is easily appreciated in Fig. 6 that the rise time (decay time) of the PL is shorter (longer) for the QW with a quasi-degenerate hh-lh excitonic ground state than for the $\mathrm{QW}$ with a hh excitonic ground state.

\section{Excitonic spin relaxation}

In this work, the spin for excitons and/or holes is defined as the third component of the total angular momentum (for electrons in III-V semiconductors, this coincides with the usual concept of spin since their orbital angular momentum is zero, "s"-states). The spin relaxation of excitons between optically active states, with spin \pm 1 , can take place in a single step, driven by the exchange interaction, or via a two-step process in which the individual constituents of the exciton, electron and hole, flip separately their spin, involving an intermediate dark state, with spin \pm 2 . The rate of exciton-spin relaxation in the latter, indirect channel is limited by the slower single-particle spin-flip rate, which usually is the electron one.

Several mechanisms are usually invoked to account for the spin relaxation of electrons, holes, and excitons. For electrons, two mechanisms are commonly considered in intrinsic semiconductors. The Elliot-Yafet mechanism $[40,41]$ is due to mixing between conduction-band and valence-band states through the $k \cdot p$ term and is only important for small gap semiconductors. The D'yakanov-Perel' (DP) mechanism [42] contemplates the spin-flip processes as a result of the asymmetry of the constituent atoms in III-V compounds with the zinc-blende structure. The anisotropic, cubic in $k$ term of the conduction band dispersion relation couples conduction-band states of opposite spins. In the case of QW's, for non-degenerate carriers, this term obtains an electron spin-flip rate proportional to the electron momentum relaxation time. In QW's the hole spin flip depends basically on valence-band mixing $[28,43]$. For $k_{\perp} \neq 0$, hole-spin relaxation occurs when the envelope functions are changed as a consequence of scattering processes. The two previous mechanisms can modify the spin state of an exciton by changing 
the individual spin of the electrons and/or holes. Furthermore, the spin relaxation of excitons between the optically active states $|+1\rangle$ and $|-1\rangle$ may occur in a single-step process driven by the exchange interaction. This mechanism was introduced in bulk semiconductors by Bir, Aronov, and Pikus (BAP) [44], and has been reformulated for QW's in Ref. [29]. This mechanism also yields a spin-flip scattering rate proportional to the exciton momentum relaxation time.

To investigate exciton-spin relaxation we measured the time evolution of the degree of circular polarization of the PL, $\wp$. The degree of circular polarization is defined, for one of the exciting helicities, as the fractional difference of the PL intensities of the two circular polarizations, $\sigma^{+}$and $\sigma^{-}$, at a given energy: i.e., for $\sigma^{+}$excitation, $\wp=\left(I^{+}-I^{-}\right) /\left(I^{+}+I^{-}\right)$. We found that, for all the samples, the time evolution of $\wp$ can be fitted to an exponential decay. Therefore, we used the decay time of $\wp, \tau_{\wp}$, as an "effective spin-relaxation time".

In the case of the GaAs/AlAs MQW we observed that exciton dynamics and its dependence on excitation energy (see Fig. 2) strongly influence the spin relaxation processes. The dependence of $\tau_{\S}$ on excitation energy is shown in Fig. 7 (diamonds) together with the cw PLE spectrum (line). The inset shows the time evolution of the polarization (points) and the best fit to a monoexponential decay (line), which obtains $\tau_{\wp}=30 \mathrm{ps,} \mathrm{exciting} \mathrm{at} 1.63 \mathrm{eV}$. The $\approx 20 \%$ larger value of $\tau_{\wp}$ at the hh excitonic peak compared to that at the subband edge demonstrates the influence of exciton wave vector on spin relaxation, and, moreover, it evidences that exchange interaction is the leading spin relaxation mechanism. Exciting at the subband continuum, large- $\boldsymbol{K}$ excitons are created from uncorrelated electron-hole pairs, while exciting at the hh exciton peak in the PLE, excitons are created resonantly with $K=\mathbf{0}$. As the long-range part of the exchange interaction is proportional to the exciton wave vector [29], the observed dependence of $\tau_{\wp}$ on excitation energy should be expected if spin relaxation were due to exchange interaction. The decrease in $\tau_{\wp}$ in the high-energy tail of the hh excitonic peak can be linked also to an increase in the long-range exchange interaction, since it has been proposed that the high-energy tail in the pseudo-absorption arises from a partial violation of the $\boldsymbol{K}$ conserving rule, which allows the creation of large- $\boldsymbol{K}$ excitons [45].

The initial behavior of the photoluminescence provides information about the removal of excitons from optically active states to dark states. We found that this behavior depends strongly on excitation and detection energy. Let us consider, first, the resonant creation of excitons with $\boldsymbol{K}=0$. Figure 8 compiles the time evolution of the $\sigma^{+}$(circles) and $\sigma^{-}$(triangles) PL for two different detection energies, $1.612 \mathrm{eV}$ (black symbols) and $1.611 \mathrm{eV}$ (gray symbols) and a lattice temperature of $5 \mathrm{~K}$. The excitation was done at $1.617 \mathrm{eV}$ with an initial carrier density of $4 \times 10^{10} \mathrm{~cm}^{-2}$. The inset shows the PL spectra excited at $1.619 \mathrm{eV}$, for a delay of 300 ps. The two arrows mark the detection energies used to measure the time evolution. In the upper left corner of the figure is also shown the laser autocorrelation signal. Two main differences can be distinguished in the figure between the black and the gray traces. One is the fast initial decay in the black $\sigma^{+}$trace, absent in the gray one. The second is the time in which circles and triangles of the same color merge, which is shorter for the gray symbols. The 


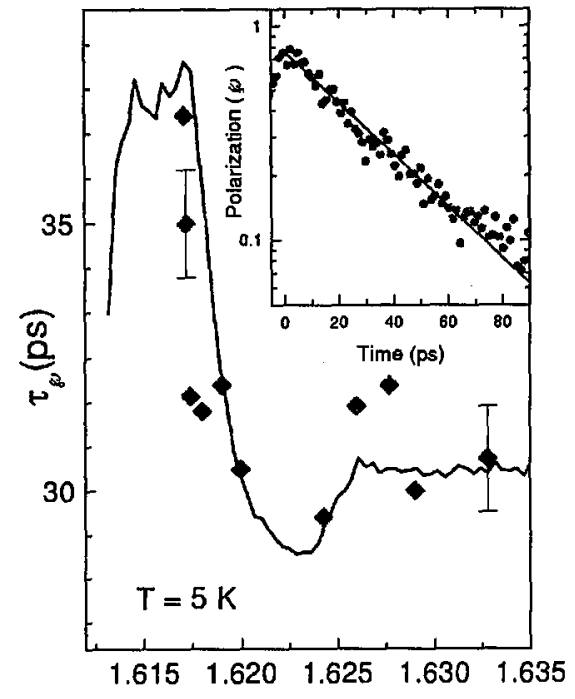

Fig. 7

Energy (eV)

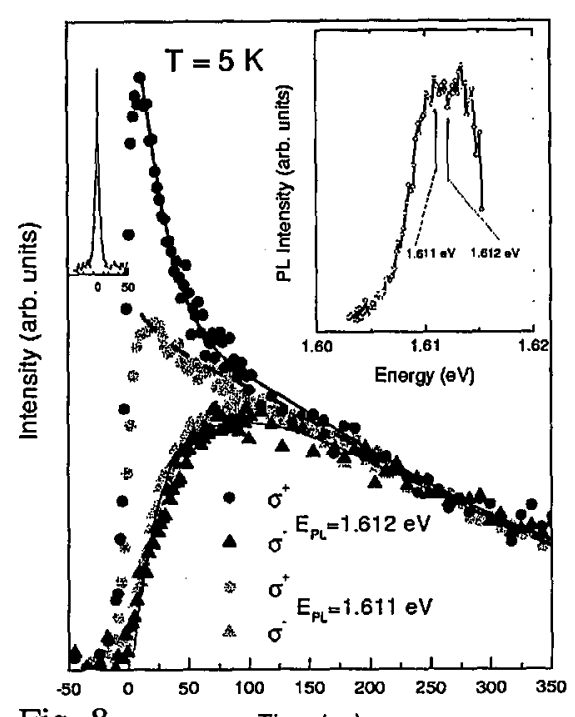

Fig. 8

Time (ps)

Fig. 7. Energy dependence of the spin-flip time $\left(\tau_{\mathfrak{p}}\right)$ (diamonds) in the intrinsic GaAs QW at $5 \mathrm{~K}$. The line shows the corresponding region of the PLE spectrum. The inset shows the time evolution of the degree of polarization at low temperature (solid points) and a fit to a monoexponential decay (line).

Fig. 8. Time evolutions of the $\sigma^{+}$(circles) and $\sigma^{-}$(triangles) photoluminescence. Black (gray) points correspond to a detection energy of $1.612(1.611) \mathrm{eV}$. The lines depict the best fits to a dynamical model, which takes into account spin flip of electrons, holes, and excitons. The inset shows a PL spectrum taken at a delay of $300 \mathrm{ps}$. The two arrows mark the detection energies used for the time traces. In the upper left corner is depicted the laser autocorrelation.

second aspect, which determines the polarization decay time will be discussed later. It has been predicted that removal of excitons from optically active states to dark states, via the spin flip of a single particle of the exciton, would give rise to a fast initial decay of the luminescence [29]. This behavior has been also observed by Vinattieri et al. in GaAs QW's under resonant excitation [18, 23]. Fitting their data with a model which takes into account spin flip of the exciton, as well as spin flip of electrons and holes individually, they have ascribed the initial decay to the spin flip of the holes. We are also able to fit our data with a similar model (lines in Fig. 8). We obtained, for the time profiles detected at $1.612 \mathrm{eV}$ (solid lines), a spin-flip time for the electrons (holes) of $\tau_{\mathrm{e}}=200 \pm 100 \mathrm{ps}\left(\tau_{\mathrm{h}}=25 \pm 8 \mathrm{ps}\right.$ ) and an exciton spin-flip time of $\tau_{\mathrm{x}}=35 \pm 10 \mathrm{ps}$. We found that the difference between black and gray traces is due to the influence of excitonic localization on the spin-flip time of holes correlated to electrons. Bastard et al. using a semi-Gaussian interface-defect model [46], have calculated that, for localized excitons on defects, a change in lateral size of $100 \AA$ produces a variation of $1 \mathrm{meV}$ in their binding energy. Moving the detection energy from 1.612 to $1.611 \mathrm{eV}$, we are sampling excitons with 
larger binding energies, and thus with stronger localization. The uncertainty in the in-plane center-of-mass momentum, $\Delta \boldsymbol{K}_{\perp}$ of these tighter-bound excitons will be larger. As $K_{\perp}=k_{\mathrm{e} \perp}+k_{\mathrm{h} \perp}$; the uncertainty in momentum of correlated electrons $\left(\Delta k_{\mathrm{e} \perp}\right)$ and holes $\left(\Delta k_{\mathrm{h} \perp}\right)$ depends on the degree of excitonic localization. Hole-spin relaxation, which is due to valence-band mixing, shows a strong dependence on $k_{\mathrm{h} \perp}$; the relaxation rate increases with increasing wave vector [43]. Therefore, keeping in mind that only $\boldsymbol{K}_{\perp} \approx 0$ excitons couple to light, for stronger localized excitons, which have a larger $\Delta k_{e \perp}$, a shorter spin-flip time of the holes is expected. As a matter of fact, the time profiles detected at $1.611 \mathrm{eV}$ (gray) are fitted with a hole and exciton spin-flip times of $\tau_{\mathrm{h}}=5 \pm 5 \mathrm{ps}$ and $\tau_{\mathrm{x}}=20 \pm 10 \mathrm{ps}$, respectively (dashed lines), keeping the rest of the times the same as those used when detecting at $1.612 \mathrm{eV}$ (black traces). Since $\tau_{\mathrm{h}}$ is of the order of our time resolution, the initial fast decay is not observed in this case.

We found that, at low temperatures, the polarization decay depends strongly on the detection energy, and that this dependence disappears on increasing the temperature. These findings are shown in Fig. 9, together with the PL spectra recorded at a delay of $300 \mathrm{ps}$, for two different temperatures: $4 \mathrm{~K}$, Fig. $9 \mathrm{a}$ and $70 \mathrm{~K}$, Fig. $9 \mathrm{~b}$. In both cases an initial carrier density of $2 \times 10^{10} \mathrm{~cm}^{-2}$ was used, and the excitation was done $20 \mathrm{meV}$ above the PL maximum. The observed dependence of $\tau_{\wp}$ is again a consequence of exciton localization. Two different reasons can be invoked to explain this behavior: one, the dependence of the scattering time on exciton localization; the second, the influence of localization on the coupling between $|+1\rangle$ and $|-1\rangle$ excitonic states.

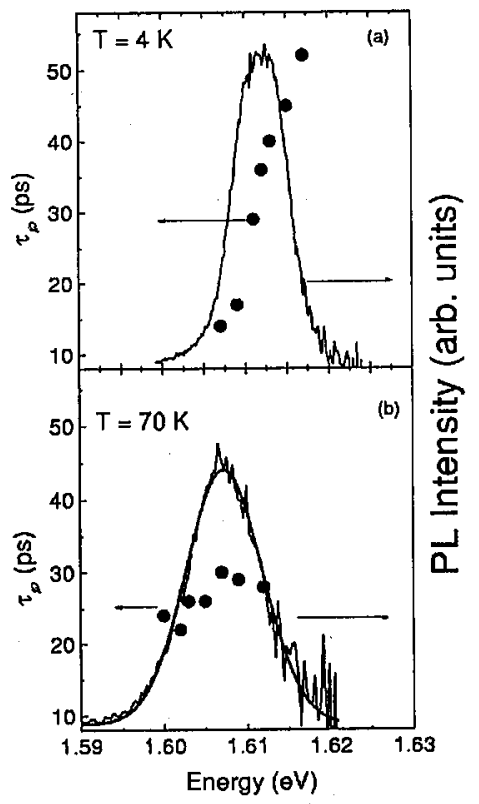

Fig. 9. Detection energy dependence of the spin-flip time ( $\left.\tau_{\mathfrak{p}}\right)$ (solid points) for two lattice temperatures (a) $4 \mathrm{~K}$ and (b) $70 \mathrm{~K}$. The lines show the PL recorded 300 ps after excitation. The thick line in (b) is a Gaussian fit of the spectrum. 
As we have mentioned above, the PL band at low temperatures is composed by the emission of localized excitons on different interface defects. The degree of localization of these excitons increases on moving towards the low-energy tail of the PL; a concomitant increase in the momentum scattering time is expected due to the decrease in the diffusion constant, and, therefore, in the mobility of the excitons [47]. This effect influences the spin relaxation caused by the DP and BAP mechanisms in a similar way, decreasing the spin-flip time in both cases since $\tau_{\wp}$ is inversely proportional to the momentum relaxation time.

The assignment of localization as the origin of the dependence of $\tau_{\wp}$ on detection energy is confirmed by the results obtained at $70 \mathrm{~K}$. At this temperature the $\tau_{\mathfrak{r}}$ dependence is strongly reduced (see Fig. 9b). The PL spectrum at $70 \mathrm{~K}$ can be fitted to a Gaussian line shape with a FWHM of $8 \pm 1 \mathrm{meV}$ (solid thick line); this value is comparable, taking into account the thermal broadening, to that obtained in very high-quality samples. Therefore we believe that the emission band at $70 \mathrm{~K}$ can be attributed to an inhomogeneous distribution of free excitons. Since the momentum scattering time of the excitons will be similar for the whole distribution, the spin-flip time is expected to be almost independent of the detection energy, as is observed.

\section{Spin splitting in a polarized exciton gas}

Figure 10 shows TR-PL spectra taken 6 ps after excitation with $\sigma^{+}$-pulses for a density of $6.5 \times 10^{10} \mathrm{~cm}^{-2}$, a temperature of $8 \mathrm{~K}$ and exciting at $1.625 \mathrm{eV}$. The gray area corresponds to the polarized $\left(\sigma^{+}\right.$, spin +1$)$ emission, while the dashed one shows the unpolarized $\left(\sigma^{-}\right.$, spin -1$)$ PL. A clear energy splitting of $\approx 4.5 \mathrm{meV}$ is clearly seen between the two peaks.

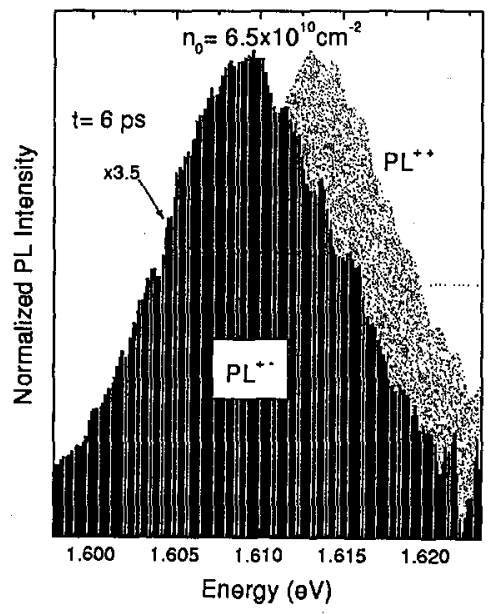

Fig. 10. Low-temperature, $8 \mathrm{~K}$, time-resolved PL spectra of the sample shown in Fig. 1 taken 6 ps after excitation with $\sigma^{+}$-polarized light at $1.625 \mathrm{eV}$. The gray (dashed) area depicts the $\sigma^{+}\left(\sigma^{-}\right)$emission. The initial carrier density is $6.5 \times 10^{10} \mathrm{~cm}^{-2}$. The $\sigma^{-}$ emission was enlarged by a factor of 3.5 . 
This splitting depends on the exciton density, which changes either because the excitation power is increased or by the annihilation of the excitons due to excitonic recombination. The dynamics of the polarized and unpolarized PL are shown in Figs. 11a and b, respectively, for an initial carrier density of $6.5 \times 10^{10} \mathrm{~cm}^{-2}$. The change in the splitting originates from a red shift of the $\sigma^{++}$-PL and a blue shift of the $\sigma^{+-}$-PL as the time delay after the excitation pulse increases, being the larger contribution than that of the unpolarized PL shift. At short times $(5 \mathrm{ps})$ the splitting amounts to $\approx 4 \mathrm{meV}$, and it vanishes for times longer than $\approx 150 \mathrm{ps}$.
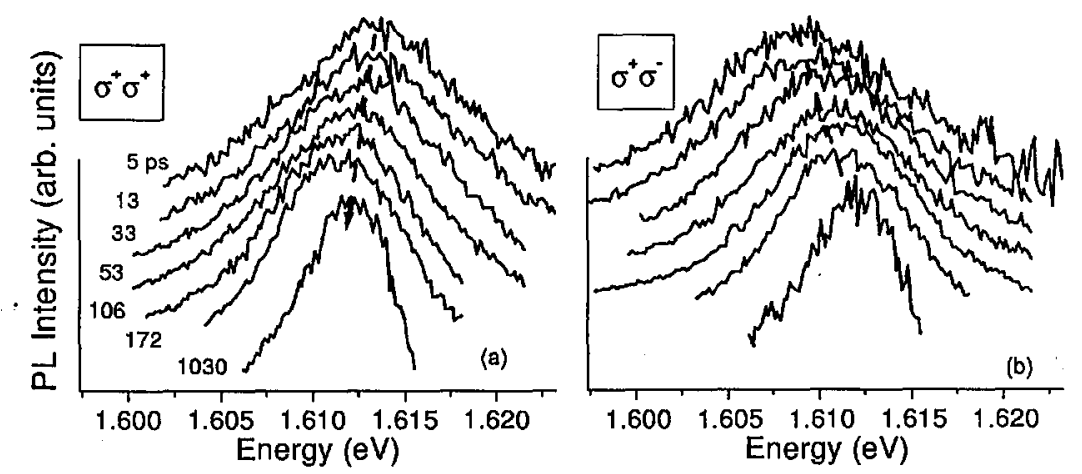

Fig. 11. Time-resolved PL spectra taken at different delays after the pulsed excitation for the $77 \AA \mathrm{GaAs} Q W$ for an initial carrier density of $6.5 \times 10^{10} \mathrm{~cm}^{-2}$. (a) Excitation with $\sigma^{+}$pulses and detection of the $\sigma^{+}$emission. (b) Excitation with $\sigma^{+}$pulses and detection of the $\sigma^{-}$emission. The lines indicate the red (blue) shift of the $\sigma^{+}\left(\sigma^{-}\right)$PL with increasing delay.
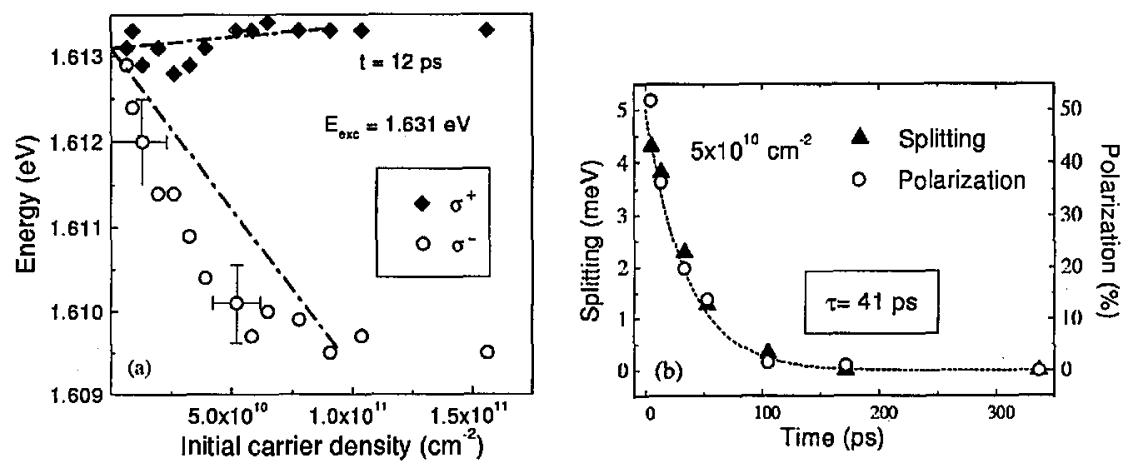

Fig. 12. (a) Energies of the co-polarized $\left(\sigma^{+}\right.$, solid points) and counter-polarized $\left(\sigma^{-}\right.$, open points) luminescence as a function of carrier density. The positions are taken $12 \mathrm{ps}$ after excitation at $1.631 \mathrm{eV}$. The lines represent the results of Eq. (2). (b) Time evolution of the PL splitting (solid points) and polarization (open points) for an initial carrier density of $5 \times 10^{10} \mathrm{~cm}^{-2}$. The line shows the best fit to an exponential decay with a time constant of $41 \mathrm{ps}$. 
Figure 12a compiles the dependence of the energy positions of the PL on the initial carrier density (open and solid points). Under the conditions presented in this figure, $12 \mathrm{ps}$ after excitation at $1.631 \mathrm{eV}$, the $\sigma^{+}$emission remains practically constant, while the $\sigma^{-}$redshifts with increasing carrier density up to $9 \times 10^{10} \mathrm{~cm}^{-2}$. The lines correspond to a model, which takes into account interexcitonic exchange interaction and screening [48], that gives the changes in the energies of the interacting \pm 1 excitons as a function of the total and the \pm 1 populations of excitons as

$$
E^{ \pm}[\mathrm{eV}]=2.214 \times 10^{-16} a[\AA] \times\left(1.515 n^{ \pm}\left[\mathrm{cm}^{-2}\right]-0.41 \pi n\left[\mathrm{~cm}^{-2}\right]\right) .
$$

For the lines in Fig. 12a, the energy of a single exciton was taken from the experimental energy of the +1 exciton at the lowest carrier density; a three-dimensional Bohr radius, $a$, of $150 \AA$ and an initial degree of polarization of $80 \%$ were used. In spite of the strong approximations used in the theory, such as neglecting the presence of dark, \pm 2 , states and assuming that the excitons are all at $K=0$, which are not borne out by the experiments, the agreement with the experiments is satisfactory.

The splitting is strongly correlated with the degree of polarization of the exciton gas, as can be observed in Fig. 12b, which depicts the time evolution of the splitting and $\wp$. This correlation is also predicted by the theory: Eq. (2) predicts that the splitting is proportional to the difference between exciton $+1\left(n^{+}\right)$and $-1\left(n^{-}\right)$populations and thus proportional to the degree of polarization $\left[\wp=\left(n^{+}-n^{-}\right) /\left(n^{+}+n^{-}\right)\right]$. When the $n^{+}$and $n^{-}$populations become similar, and therefore $\wp$ approaches zero, the splitting disappears as a consequence of the convergence of the \pm 1 excitons towards the same energy. The time decay of $\wp$ originates from the excitonic spin-flip processes described in Sec. 3, which are mainly driven by intraexcitonic exchange interaction [16].

\section{Spin dynamics of an optically pumped electron gas}

Exciting a $p$-type GaAs quantum well below the light-hole resonance, electrons with almost purely one spin component are photocreated. Figure 13 depicts the PLE spectra of a $30 \AA$, [100]-oriented QW at $2 \mathrm{~K}$, exciting with $\sigma^{+}$-polarized light recorded at the tail of the PL. The onset of the absorption due to the hh transition is clearly seen as a peak in the $\sigma^{+} \sigma^{+}$spectrum at $1.7 \mathrm{eV}$, while the one corresponding to the lh transition is dominant in the $\sigma^{+} \sigma^{-}$spectrum at $1.738 \mathrm{eV}$. As can be deduced from this figure, the sample shows a high degree of optical alignment, indicating a long spin-flip relaxation time for the photoexcited electrons.

Using these $p$-type samples, we can easily investigate spin alignment effects in the conduction band: electrons with an unbalanced population of the two spin components, created under circularly polarized excitation, recombine with non-polarized holes which mostly originate from doping. Therefore the spin relaxation of the electrons can be obtained from the difference of the time evolution of the two orthogonally polarized emissions. The spin-relaxation time of photogenerated electrons in $p$-doped QW's has been calculated by Maialle and Degani (Ref. [34]). They have found that, for the mechanism of exchange interaction, the 


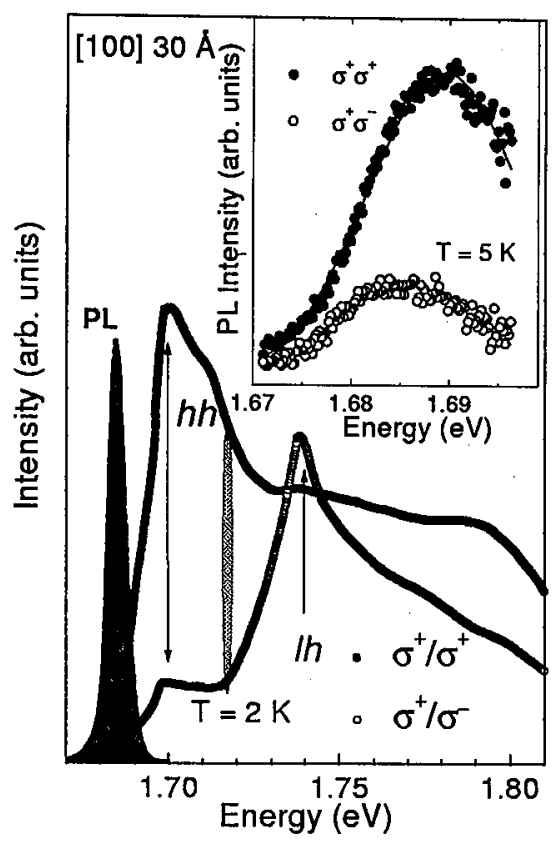

Fig. 13. $\mathrm{PL}$ (shaded area) and excitation spectra of a $30 \AA$ thick $p$-type modulation doped ( $p=3 \times 10^{11} \mathrm{~cm}^{-2}$ ) QW measured for aligned (solid points) and crossed (open symbols) polarization of the exciting/emitted light. The hatched area indicates the energy used for excitation on the time-resolved experiments. The inset displays the co-polarized and counter-polarized components of the PL spectra, measured 15 ps after a $\sigma^{+}$-polarized pulsed excitation with a mean laser power of $20 \mathrm{~mW}$ and excitation energy of $1.717 \mathrm{eV}$. Bath temperature $5 \mathrm{~K}$.

spin mixing of the valence hole is not important due to a compensation between the enlargement of the hole density of states and a spin-mixing induced decrease in the exchange strength.

In an ideal sample, using the estimated hole Fermi energy of $2 \mathrm{meV}$, and assuming a ratio of six between the hole and electron effective masses, the circularly polarized luminescence spectra are expected to directly reflect the distributions of electrons with different spin components in an energy range up to $\approx 12 \mathrm{meV}$ above the conduction-band edge. However, as shown in the inset of Fig. 13, the spectra measured at 15 ps after a $20 \mathrm{~mW}$ excitation cover a somehow wider spectral range. We assume that even these spectra probe only the properties of the electron gas since the investigated sample shows an appreciable spectral broadening already under low-power $\mathrm{cw}$ excitation (half width of the luminescence $\approx 10 \mathrm{meV}$ ). The inset represents the $\sigma^{+}$and $\sigma^{-}$components of the luminescence spectra excited with $\sigma^{+}$pulses at $1.717 \mathrm{eV}$. These spectra clearly show the difference in the occupation of electronic states with opposite spins. This difference, which vanishes at longer delay times, leads to the energy difference between the positions of the maxima in the $\sigma^{+}$and $\sigma^{-}$luminescence spectra. 


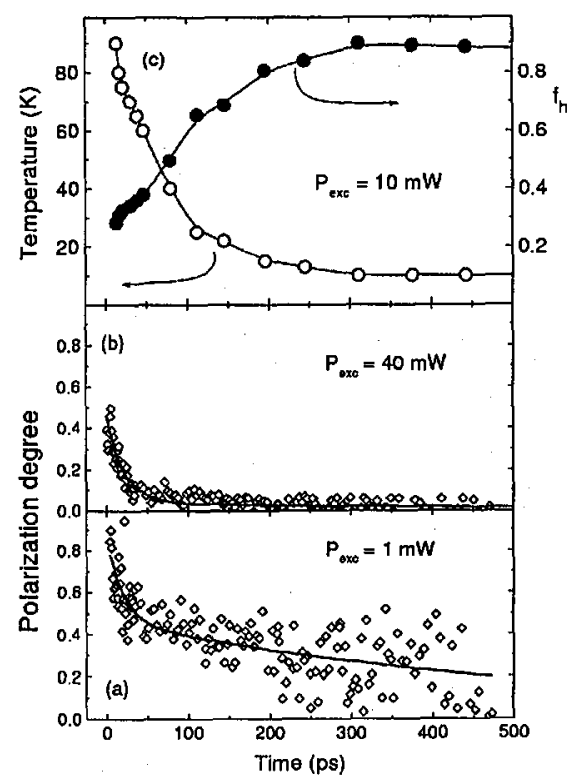

Fig. 14. (a) Time evolution of the polarization degree of the sample shown in Fig. 13 for $1 \mathrm{~mW}$ mean laser power. (b) Same as (a) for a laser power of $40 \mathrm{~mW}$. The lines represent the best fits according to the sum of two exponential decays: $A \exp \left(-t / \tau_{1}\right)+$ $B \exp \left(-t / \tau_{2}\right)$. (c) Time evolution of the carrier temperature (open circles), for a mean laser power of $10 \mathrm{~mW}$, obtained from the fits of the time-resolved spectra using Eq. (3). The solid symbols depict the time evolution of the number of occupied hole states at the top of the valence band $\left(E_{\mathrm{h}}=0\right)$.

The most conspicuous finding is illustrated in Fig. 14: parts (a) and (b) depict the time evolution of the polarization degree, $\wp$, for two different powers of the exciting light. We found that the decay of spin polarization of the electron gas depends very much on the intensity of the laser excitation. The decay of the degree of luminescence polarization, $\wp$, measured at $T=8 \mathrm{~K}$ can be well reproduced by a sum of two exponential decays with two distinct characteristic times of $\tau_{1}=20 \mathrm{ps}$ and $\tau_{2}=550 \mathrm{ps}$. The amplitude of the fast component vanishes at low excitation power, whereas this fast process almost completely determines the electron spin depolarization at the highest level of laser excitation. It is well known that for any mechanism of electron spin relaxation [42], the probability of spin flip transitions increases as a function of the electron $k$-vector. Therefore, the increase in the rate of spin relaxation as a function of the laser power may result partially from a larger electron kinetic energy caused either by an increase in the effective electron temperature or in the initial electron concentration. However, this simple reasoning hardly explains our data. From an analysis of the time evolution of the luminescence spectra, we estimated an increase in the mean kinetic energy of the electron gas of only $4 \mathrm{meV}$ at the highest laser power. This amount is not sufficient to reduce the spin relaxation time down to $20 \mathrm{ps}$, since at low excitation powers but high lattice temperatures $(k T \approx 3.44 \mathrm{meV})$ we still observe a relatively long spin relaxation time $(\approx 80 \mathrm{ps})$. 
We simulated the measured spectra at different times after the excitation, $I^{+(-)}(\hbar \omega)$, by the broadened convolution of the Fermi-Dirac statistics for non-polarized gas of holes and two spin components of the 2D electron gas (2DEG), assuming the conservation of $k$-selection rules

$$
\begin{aligned}
& I^{+(-)}\left(\hbar \omega-E_{\mathrm{g}}\right)=I^{+(-)}\left(E_{\mathrm{e}}+E_{\mathrm{h}}\right) \\
& =A \int_{0}^{\infty} f_{E_{\mathrm{e}}^{\prime}}^{+(-)} f_{E_{\mathrm{h}}^{\prime}} G_{\Gamma}\left(E_{\mathrm{e}}-E_{\mathrm{e}}^{\prime}\right) \delta\left(k_{\mathrm{e}}^{\prime}-k_{\mathrm{h}}^{\prime}\right) \mathrm{d} E_{\mathrm{e}}^{\prime} .
\end{aligned}
$$

Here $E_{\mathrm{g}}$ is the energy gap; e (h) stands for electrons (holes); $I^{+(-)}$denotes the intensity of $\sigma^{+}\left(\sigma^{-}\right)$luminescence, $G_{\Gamma}(x)$ is a Gaussian broadening function with a broadening parameter, $\Gamma$ of $7 \mathrm{meV}$, chosen to reproduce the low-temperature $(4 \mathrm{~K})$, low-power $\left(1 \mathrm{~mW} / \mathrm{cm}^{2}\right) \mathrm{cw}$ spectra, $E_{\mathrm{e}(\mathrm{h})}=\hbar k_{\mathrm{e}(\mathrm{h})}^{2} / 2 m_{\mathrm{e}(\mathrm{h})}$ is the electron (hole) energy, where we assumed $m_{\mathrm{e}}=0.075 \mathrm{~m}_{0}$ and $m_{\mathrm{e}} / m_{\mathrm{h}}=0.18$.

We will concentrate on the data taken with an excitation power of $10 \mathrm{~mW}$. An analysis of pairs of $\sigma^{+}$and $\sigma^{-}$PL spectra leads us first to conclude that each component is well described assuming a common temperature for the two electron spin components (and for holes), but different values of the chemical potential. Each spin component of the electron gas can be qualitatively characterized by its own Fermi distribution, each one with different chemical potential but both with very similar temperatures. To fit our measurements, we assumed that the time evolution of the total electron concentration follows the decay of the total luminescence intensity observed at sufficiently long delay times. The initial electron concentration was found by self-consistent fitting of several spectra measured at long delay times. Hole concentration was assumed to be $n_{\mathrm{h}}=n^{+}+n^{-}+n_{\mathrm{h}}^{0}$, where $n_{\mathrm{h}}^{0}$ originates from modulation doping. Electron concentrations $n^{+(-)}$, which define the corresponding chemical potentials, were determined from the experiment assuming $n^{+} / n^{-}$to be equal to the ratio of the integrated intensities of the $\sigma^{+}$ and $\sigma^{-}$PL. Finally, a given pair of $\sigma^{+}$and $\sigma^{-}$spectra was fitted with only two parameters: carrier temperature and a proportionality factor, $A$, which was found to be common for all the simulated spectra, within experimental error.

The obtained time evolution of carrier temperature, is shown in Fig. 14c (open circles). The carrier temperature rises up to $\approx 100 \mathrm{~K}$ just after the laser pulse. This fact, in conjunction with the measurements as a function of lattice temperature (see Sec. 3), accounts for the fast depolarization of electronic spins, induced by the laser power. Our results confirm the high efficiency of carrier-carrier interaction in establishing a common temperature for electrons and holes. Cold before excitation, the gas of holes becomes non-degenerate almost immediately after the laser pulse. This non-degenerate character of the hole gas is illustrated in Fig. 14c (solid circles), where the number of occupied hole states at the top of the valence band is plotted as a function of time.

We therefore conclude that fast spin depolarization in our structures is driven by the non-degenerate character of carrier distribution and not exclusively by the increase in the electron kinetic energy. Such behavior can be understood in terms of the BAP mechanism of the electron spin relaxation, but it is hardly accounted for by the DP processes whose efficiency is directly related to the electron kinetic energy. As can be deduced from our previous discussion, non-degenerate carrier 
distributions favor the efficiency of spin-flip electron scattering via the exchange interaction with holes, in contrast, the available number of scattering configurations is appreciably reduced for the degenerate systems.

\section{Summary}

We showed that the study of carrier and exciton dynamics in two-dimensional semiconductors is a very active field of research with many points open to future investigations. Spin-flip processes for excitons in $2 \mathrm{D}$ systems are dominated by intraexcitonic exchange (BAP), although spin flip of the individual fermions forming the exciton is responsible for fast decays observed in the TR-PL emission. The role of localization on the spin-flip dynamics was clarified. Interexcitonic exchange is shown to be responsible for a splitting of the excitons in a polarized gas. In the case of a 2DEG, when this gas is created polarized by means of optical orientation and becomes dense, strong effects, nonlinear in the excitation power, are observed in the polarization of the emission, which originates from the non-degenerate character of the carrier distribution at short times after the laser excitation. An optically aligned, spin-polarized electron gas can be well described by two separate Fermi-Dirac distribution functions, one for each spin component, with common temperature but different chemical potentials.

\section{Acknowledgments}

This work would not have been possible without the collaboration with many people, especially with: L. Muñoz, E. Pérez, J. Fernández-Rossier, and C. Tejedor, who made a great work in the study of excitons. The studies of 2DEG were made in collaboration with M. Potemski and L. Gravier; M.D. Martín contributed considerably to these studies. The samples were kindly provided by K. Ploog and K.M. Lau. This research has been partially supported by the Fundación Ramón Areces, the Spanish DGICYT under contract PB96-0085 and CAM 7N/0026/1998.

\section{References}

[1] J. Feldmann, G. Peter, E.O. Gobel, P. Dawson, K. Moore, C. Foxon, R.J. Elliott, Phys. Rev. Lett. 59, 2337 (1987).

[2] J. Kusano, Y. Segawa, Y. Aoyagi, S. Namba, H. Okamoto, Phys. Rev. B 40, 1685 (1989).

[3] T.C. Damen, J. Shah, D.Y. Oberli, D.S. Chemla, J.E. Cunningham, J.M. Kuo, Phys. Rev. B 42, 7434 (1990).

[4] R. Eccleston, R. Strobel, W.W. Ruhle, B.F. Feuerbacher, K. Ploog, Phys. Rev. B 44, 1395 (1991).

[5] B. Deveaud, F. Clerot, N. Roy, K. Satzke, B. Sermage, D.S. Katzer, Phys. Rev. Lett. 67, 2355 (1991).

[6] P. Roussignol, C. Delalande, A. Vinattieri, L. Carraresi, M. Colocci, Phys. Rev. $B$ 45, 6965 (1992).

[7] P.W.M. Blom, P.J. van Halle, C. Smit, J.P. Cuypers, J.H. Wolter, Phys. Rev. Lett. 71,3878 (1993). 
[8] B. Deveaud, F. Clerot, B. Sermage, C. Dumas, D.S. Katzer, in: Optical Phenomena in Semiconductor Structures of Reduced Dimension, Eds. D.J. Lockwood, A. Pinczuk, Vol. 248, Kluwer Academic Publ. B.V., The Netherlands 1993, p. 129.

[9] B. Dareys, X. Marie, T. Amand, B. Baylac, J. Barreau, I. Razdobreev, M. Brousseau, D. Dunstan, Solid State Commun. 90, 237 (1994).

[10] A. Vinatteri, J. Shah, T.C. Damen, D.S. Kim, L.N. Pfeiffer, M.Z. Maialle, L.J. Sham, Phys. Rev. B 50, 10868 (1994).

[11] T. Amand, X. Marie, B. Baylac, B. Dareys, J. Barrau, M. Brousseau, D.J. Dunstan, R. Planel, Phys. Lett. A 193, 105 (1994).

[12] L. Muñoz, E. Pérez, V. Bellani, S. Zimmermann, L. Viña, K. Ploog, E.S. Koteles, K.M. Lau, J. Opt. Soc. Am. B 13, 994 (1996).

[13] W. Hanle, Z. Phys. 30, 93 (1924).

[14] H.-S. Chao, K.S. Wong, R.R. Alfano, H. Unlu, H. Morkoc, J. Soc. Photo-Opt. Instrum. Eng. 942, 215 (1988).

[15] M.R. Freeman, D.D. Awschalom, J.M. Hong, L.L. Chang, Phys. Rev. Lett. 64, $2430(1990)$.

[16] T.C. Damen, L. Viña, J.E. Cunningham, J. Shah, Phys. Rev. Lett. 67, 3432 (1991).

[17] P. Roussignol, P. Rolland, R. Ferreira, C. Delalande, G. Bastard, A. Vinattieri, J. Martinez-Pastor, L. Carraresi, M. Colocci, J.F. Palmier, B. Etienne, Phys. Rev. $B$ 46, 7292 (1992).

[18] A. Vinattieri, J. Shah, T.C. Damen, D.S. Kim, L.N. Pfeiffer, L.J. Sham, Solid State Commun. 88, 189 (1993).

[19] I. Bar-Joseph, S. Bar-Ad, O. Carmel, Y. Levinson, in Ref. [8], p. 173.

[20] L. Muñoz, E. Pérez, L. Viña, K. Ploog, Phys. Rev. B 51, 4247 (1995).

[21] T. Amand, D. Robart, X. Marie, M. Brousseau, P.L. Jeune, J. Barrau, Phys. Rev. $B$ 55, 9880 (1997).

[22] H. Nickolaus, H.-J. Wuensche, F. Henneberger, Phys. Rev. Lett. 81, 2586 (1998).

[23] A. Vinattieri, J. Shah, T.C. Damen, K.W. Goossen, L.N. Pfeiffer, M.Z. Maialle, L.J. Sham, Appl. Phys. Lett. 63, 3164 (1993).

[24] E.L. Ivchenko, P.S. Kopev, V.P. Kochereshko, I.N. Uraltsev, D.R. Yakovlev, JETP Lett. 47, 486 (1988).

[25] J. Wagner, H. Schneider, D. Richards, A. Fischer, K. Ploog, Phys. Rev. B 47, 4786 (1993).

[26] R. Ferreira, P. Roussignol, P. Rolland, G. Bastard, C. Delalande, A. Vinattieri, G. Weimann, J. Phys. II, Colloq. C5 3, 175 (1993).

[27] L.J. Sham, J. Phys. (Paris), Colloq. C5 48, 381 (1987).

[28] T. Uenoyama, L.J. Sham, Phys. Rev. B 42, 7114 (1990).

[29] M.Z. Maialle, E.A. de Andrada è Silva, L.J. Sham, Phys. Rev. B 47, 15776 (1993).

[30] E.A. de Andrada e Silva, G.C.L. Rocca, Phys. Rev. B 56, 9259 (1997).

[31] M.I. D’yakonov, V.Y. Kachorovskii, Sov. Phys.-Semicond. 20, 110 (1986).

[32] G. Bastard, R. Ferreira, Surf. Sci. 267, 335 (1992).

[33] R. Ferreira, G. Bastard, Solid State Electron. 37, 851 (1994).

[34] M.Z. Maialle, M.H. Degani, Phys. Rev. B 55, 13771 (1997). 
[35] L. Gravier, M. Potemski, K. Ploog, in: Proc. 22nd Int. Conf. on the Physics of Semiconductors, Vancouver 1994, Ed. D.J. Lockwood, World Scientific, Singapore 1995, p. 1213.

[36] B.D.C. Bertolet, J. Hsu, K.M. Lau, Appl. Phys. Lett. 53, 2501 (1988).

[37] E.P. O'Reilly, Semicond. Sci. Technol. 4, 121 (1989).

[38] L. Viña, L. Muñoz, N. Mestres, E.S. Koteles, D.C. Bertolet, K.M. Lau, Philos. Mag. B 70, 397 (1994).

[39] L. Viña, L. Muñoz, N. Mestres, E.S. Koteles, A. Ghiti, E.P. O’Reilly, D.C. Bertolet, K.M. Lau, Phys. Rev. B 47, 13926 (1993).

[40] R.J. Elliot, Phys. Rev. 98, 1654 (1955).

[41] Y. Yaffet, Solid State Phys. 14, 1 (1963).

[42] M.I. D'yakonov, V.I. Perel', Sov. Phys.-JETP 33, 1053 (1971).

[43] R. Ferreira, G. Bastard, Phys. Rev. B 43, 9687 (1991).

[44] G.L. Bir, A.G. Aronov, G.E. Pikus, Sov. Phys.-JETP 42, 705 (1976).

[45] R.F. Schnabel, R. Zimmermann, B. Bimberg, H. Nickel, R. Lösch, W. Schlapp, Phys. Rev. B 46, 9873 (1992).

[46] G. Bastard, C. Delalande, M.H. Meynadier, P.M. Frijlink, M. Voos, Phys. Rev. B 29, 7402 (1984).

[47] J. Hegarty, L. Goldner, M.D. Sturge, Phys. Rev. B 30, 7346 (1984).

[48] J. Fernandez-Rossier, C. Tejedor, L. Muñoz, L. Viña, Phys. Rev. B 54, 11582 (1996). 